\title{
Level of systolic blood pressure within the normal range and risk of cardiovascular events in the absence of risk factors in
}

\section{Chinese}

\author{
Chunpeng Ji $\mathbb{D}^{1,7}$, Na Wang $\mathbb{D}^{2,7}$, Jihong Shi $\mathbb{D}^{1}$, Zhe Huang $\mathbb{D}^{1}$, Shuohua Chen $\mathbb{D}^{3}$, Guodong Wang $\mathbb{D}^{3}$, Shouling Wu (D) ${ }^{1 凶}$ and \\ Jost B. Jonas (iD) $4,5,6 \bowtie$
}

(C) The Author(s) 2021

The risk of cardiovascular disease (CVD) at currently defined normal systolic blood pressure (SBP) levels in individuals without CVD risk factors is not well examined. We evaluated whether higher systolic blood pressure within the range considered normal is associated with a higher CVD risk in Chinese without traditional CVD risk factors. The community-based study included 25,529 individuals (mean age:47.3 \pm 12.3 years;range:18-95 years) with a baseline SBP of 90-129 mmHg, who were free of CVD and traditional CVD risk factors, and who were re-examined in biennial intervals. During a mean follow-up of $10.6 \pm 1.49$ years (maximum. 11.5 years), 847 CVD events occurred. CVD incidence per 1000 person-years increased with higher baseline SBP levels (SBP,90-99 mmHg:1.45;100-109 mmHg:2.15;110-119 mmHg:3.06; and 120-129 mmHg:3.80). After adjusting for CVD risk factors, the categorical Cox regression suggested that the CVD risk was not statistically significant for study participants with a baseline SBP level of $100-109 \mathrm{mmHg}, 110-119 \mathrm{mmHg}$, and $120-129 \mathrm{mmHg}$ compared with those with a baseline SBP level of $90-99 \mathrm{mmHg}$. If CVD risk factors including blood pressure categories which developed during follow-up were included in a time-dependent Cox regression analysis, the normal baseline SBP was still not associated with incident CVDs. A SBP between 90 and $129 \mathrm{mmHg}$ was not associated with an increased CVD risk in a healthy population.

Journal of Human Hypertension (2022) 36:933-939; https://doi.org/10.1038/s41371-021-00598-1

\section{INTRODUCTION}

As an important risk factor for cardiovascular diseases (CVDs), high blood pressure (BP) has been a leading cause of disability and death $[1,2]$. In the Global Burden of Diseases Study 2019, high systolic BP (SBP), defined by a theoretical minimum-risk exposure level of $110-115 \mathrm{mmHg}$, accounted for 10.8 million deaths $(19.2 \%$ of all deaths) [1, 3]. With an improvement in BP control, more than $60 \%$ of incident CVD events occurred in the normotensive population (SBP/diastolic BP [DBP] $<140 / 90 \mathrm{mmHg}$ ) [4]. This also led to a shift in the focus of BP-related research.

A recent meta-analysis including 61 cohort studies showed that the CVD risk increased starting from a relatively low BP level (SBP/ DBP: $115 / 75 \mathrm{mmHg}$ ) [5]. The SBP intervention trial (SPRINT) found that intensive antihypertensive treatment (lowering SBP/DBP to $<120 / 80 \mathrm{mmHg}$ ), as compared with standard antihypertensive treatment with a lowering of the SBP/DBP to $<140 / 90 \mathrm{mmHg}$, reduced the risk of CVD and all-cause death [6]. Based on the results of these and other studies, the 2017 American Heart Association (AHA)/American College of Cardiology (ACC) guidelines reduced the SBP level defining hypertension from 140 to 130 $\mathrm{mmHg}$ [7]. It has remained unclear whether the association between the SBP level and the CVD risk is valid also among healthy normotensive adults without traditional cardiovascular risk factors. The multi-ethnic study of atherosclerosis (MESA) recently reported that in 1457 normotensive participants without conventional cardiovascular risk factors, the CVD risk increased during an average follow-up of 14.5 years by $53 \%$ for every 10 $\mathrm{mmHg}$ increase in the baseline SBP, ranging between 90 and 129 $\mathrm{mmHg}$ [8]. It suggested a dose-effect rather than a J-shaped or U-shaped relationship between the SBP level and CVD risk in a healthy population. Since such results are of clinical and general importance and since the CVD incidence and its risk factors differ between regions and societies, we performed this study to reinvestigate the association between the baseline SBP level within the normal range and the CVD risk in a normotensive population without conventional cardiovascular risk factors in China.

\section{METHODS}

The Kailuan study (registration number: ChiCTR-TNC-1100148) is a prospective community-based cohort study that was performed in the community of Kailuan in the industrial city of Tangshan in the Chinese province of Hebei [9-11]. The Ethics Committees of Kailuan General Hospital confirmed that the study followed the guidelines of the Helsinki Declaration and approved it. All participants signed a written informed

\footnotetext{
${ }^{1}$ Department of Cardiology, Kailuan General Hospital, North China University of Science and Technology, Tangshan, China. ${ }^{2}$ Department of Cardiology, The Second Hospital of Qinhuangdao, Qinhuangdao, China. ${ }^{3}$ Health Care Center, Kailuan Medical group, Tangshan, China. ${ }^{4}$ Department of Ophthalmology, Medical Faculty Mannheim of the Heidelberg University, Mannheim, Germany. ${ }^{5}$ Institute of Molecular and Clinical Ophthalmology Basel, Basel, Switzerland. ${ }^{6}$ Privatpraxis Prof Jonas und Dr Panda-Jonas, Heidelberg, Germany. ${ }^{7}$ These authors contributed equally: Chunpeng Ji, Na Wang. ${ }^{{ }}$email: drwusl@163.com; Jost.Jonas@medma.uni-heidelberg.de
} 
consent. The study participants were employees and retirees of the Kailuan Group Company. The latter is a coal mining industry in Tangshan. At baseline of the study between June 2006 and October 2007, we examined the study population of 101,510 individuals $(81,110$ men) with an age ranging between 18 and 98 years. All participants underwent reexaminations in the clinical examination rooms in 2-year intervals. The examinations included an interview with standardized questions on demographic, socioeconomic and clinical parameters, determination of anthropometric parameters, measurement of $\mathrm{BP}$, biochemical analysis of blood samples, and other measures. Mercury sphygmomanometers were used to measure the BP of the left arm with a cuff of appropriate size following the recommended standard procedures. After the participants had rested in a chair for at least $5 \mathrm{~min}$, we took the measurements at $5 \mathrm{~min}$ intervals. We used the average of three readings for further data analysis. The eGFR was calculated using the Chronic Kidney Disease Epidemiology Collaboration (CKD-EPI) formula [12]

For the present investigations, we included all participants of the Kailuan Study with a normal SBP $(90-129 \mathrm{mmHg})$ and without traditional CVD risk factors at the baseline visit in 2006-2007. We excluded participants with missing values of SBP, serum concentrations of low-density lipoprotein cholesterol (LDL-C), high-density lipoprotein cholesterol (HDL-C) and glucose $(n=1557)$, individuals with a SBP $<90 \mathrm{mmHg}(n=318)$ or a $\mathrm{SBP} \geq 130 \mathrm{mmHg}(n=51,156)$, persons taking medications against hypertension $(n=1762)$, individuals with dyslipidemia (defined by a LDL-C level $\geq 160 \mathrm{mg} / \mathrm{dL}$, HDL-C level $<40 \mathrm{mg} / \mathrm{dL}$, or reported use of cholesterollowering medications, $n=4455$ ), patients with diabetes (glucose level $\geq 126 \mathrm{mg} / \mathrm{dL}$ or use of blood glucose-lowering medications, $n=2269$ ), current smokers $(n=14,152)$, and individuals with a history of CVD $(n=$ 312). It left a total of 25,529 participants, who were followed up till December 31st, 2017 or up to the time at which a CVD event including myocardial infarction and stroke, or death occurred, whichever came first. We defined CVD risk factors based on conventional categorical CVD risk factors as published previously [13-15].

The main outcome parameter was the incident CVD event including myocardial infarction, heart failure, cerebral infarction, and cerebral hemorrhage. We defined an CVD event as described previously [10]. All study participants were linked to the Municipal Social Insurance Institution and the Hospital Discharge Register which allowed the detection of an incident CVD. To identify potential additional study participants with CVD events, we reviewed the discharge lists from the 11 Kailuan hospitals during the study period from 2006 to 2017, and we asked the study participants at each re-examination about a previous CVD event. For all suspected CVD events, three experienced masked physicians reviewed the medical records and adjudicated. Incident myocardial infarction was diagnosed according to the criteria of the World Health Organization on the basis of clinical symptoms, changes in the serum concentrations of cardiac enzymes and/or biomarkers, and electrocardiogram results [16, 17]. The diagnosis of heart failure was based on clinical symptoms and objective evidence (such as abnormalities of echocardiography, chest radiographs, B-type natriuretic peptide, etc.) [18]. Stroke was diagnosed according to the World Health Organization criteria [19].

\section{Statistical analysis}

We divided the study population into four groups based on their SBP levels, namely $90-99 \mathrm{mmHg}, 100-109 \mathrm{mmHg}, 110-119 \mathrm{mmHg}$, and $120-129 \mathrm{mmHg}$. The incidence density of CVD events was calculated as number of cases divided by person-years. To examine the association between the baseline SBP (continuous) and incident CVD, we performed a restricted cubic spline interpolation with a reference value of a SBP of 110 $\mathrm{mmHg}$ allowing for three knots, which were selected based on Harrell's recommended percentiles at SBP values of $98.7 \mathrm{mmHg}$ (5th percentile), $116.7 \mathrm{mmHg}$ (50th percentile), and $126 \mathrm{mmHg}$ (95th percentile) [20]. This restricted cubic spline interpolation was adjusted for age, sex, alcohol consumption status (never and past, or current (i.e., $\geq$ once/day)), ever smoking (yes/no), education level (elementary school, high school, or above), physical exercise (none, occasionally, or frequently (i.e., $\geq$ once/ week)), prediabetes (yes/no), family history of CVD (yes/no), DBP, body mass index, estimated glomerular filtration rate (eGFR), and serum concentrations of total cholesterol, high-density lipoproteins- $C$, glucose serum concentration, uric acid, and C-reactive protein.

The Cox regression was used to estimate hazard ratios (HRs) and 95\% confidence intervals (Cls) for CVD in the different SBP groups with an SBP level of $90-99 \mathrm{mmHg}$ as the reference. Model 1 was unadjusted, model 2 was adjusted for age and sex, model 3 was further adjusted for the other covariates mentioned above, and model 4 was a competing risk model of death which adjusted for all the confounders in model 3. We further examined the interaction between the age group (median grouping) and the SBP category, and between sex and the SBP category in the Cox regression models. We tested the proportional hazards assumption graphically and verified it using the Schoenfeld residual method. The assumption for proportionality was not violated.

We additionally performed sensitivity analyses after excluding participants with a DBP $\geq 80 \mathrm{mmHg}(n=11,897)$ or after excluding individuals who met the predefined categorical cut points to be free of CVD risk factors, but who had risk factor values in the upper normal range, i.e., (1) LDL-C level $\geq 130 \mathrm{mg} / \mathrm{dL}$, or women with an HDL-C level $<50 \mathrm{mg} / \mathrm{dL}$ ( $n=$ 3164 ), and (2) blood glucose concentrations $\geq 100 \mathrm{mg} / \mathrm{dL}(n=4122)$.

To examine whether changes in SBP and changes in the CVD risk factor parameters during the follow-up had an effect on the results, we eventually performed a time-dependent Cox regression analysis using the measurements of SBP and other CVD risk factors during the follow-up at visit \#2 (2008-2009), visit \#3 (2010-2011), visit \#4 (2012-2013), visit \#5 (2014-2015), and visit \#6 (2016-2017). The participants were divided into five groups based on their SBP levels at each follow-up, namely 90-99 $\mathrm{mmHg}, 100-109,110-119 \mathrm{mmHg}, 120-129 \mathrm{mmHg}$, and $\geq 130 \mathrm{mmHg}$. In the time-dependent Cox regression, the follow-up time for each individual was divided into different short-time windows (each follow-up interval), a specific HR was calculated for each time window, and the weighted average of these window-specific HRs was determined. The traditional and time-dependent Cox regression analyses were performed again with a SBP level of $90-109 \mathrm{mmHg}$ as the reference. We also calculated the rate of progression to systolic hypertension and the prevalence of CVD in different SBP groups at baseline. The SAS software (version 9.2, SAS Institute, Cary, NC, USA) was used for the statistical analyses. All statistical tests were twosided, and a $P$ value $<0.05$ was considered statistically significant.

\section{RESULTS}

The study included 25,529 participants (15,297 (59.9\%) men) with a mean age of $47.3 \pm 12.3$ (range: 18-95) years. The mean baseline SBP and mean baseline DBP were $114 \pm 9.17 \mathrm{mmHg}$ and $75.8 \pm$ $7.50 \mathrm{mmHg}$, respectively. During the study period, the mean SBP and DBP were $119 \pm 15.8 \mathrm{mmHg}$ and $79.0 \pm 9.47 \mathrm{mmHg}$, respectively, at 2 years after baseline, $121 \pm 15.2 \mathrm{mmHg}$ and $79.9 \pm 9.42$ $\mathrm{mmHg}$, respectively, at 4 years after baseline, $122 \pm 15.7 \mathrm{mmHg}$ and $79.8 \pm 9.32 \mathrm{mmHg}$, respectively, at 6 years after baseline, 128 $\pm 16.8 \mathrm{mmHg}$ and $78.7 \pm 10.0 \mathrm{mmHg}$, respectively, at 8 years after baseline, and $130 \pm 16.9 \mathrm{mmHg}$ and $77.8 \pm 10.3 \mathrm{mmHg}$, respectively, at 10 years after baseline.

Analyzing the baseline examination data in univariate analysis, an increasing baseline SBP was associated with older age $(P<$ $0.001)$, higher proportion of men $(P<0.001)$, lower level of education $(P<0.001)$, higher prevalence of ever smoking $(P<$ $0.001)$, higher body mass index $(P<0.001)$, higher serum concentrations of total cholesterol $(P<0.001)$, low-density lipoproteins- $C(P<0.001)$, glucose $(P<0.001)$, C-reactive protein $(P<$ $0.001)$ and uric acid $(P<0.001)$, higher prevalence of prediabetes, and lower eGFR (Table 1).

During a mean follow-up of $10.6 \pm 1.49$ years (range: $0.04-11.5$ years), 847 CVD events occurred. The rate of incident CVD events per 1000 person-years increased with increasing baseline SBP levels, with the baseline SBP levels of $90-99 \mathrm{mmHg}, 100-109$ $\mathrm{mmHg}, 110-119 \mathrm{mmHg}, 120-129 \mathrm{mmHg}$ having incidence of 1.45 , $2.15,3.06$, and 3.80 , respectively (Table 1 ).

After adjusting for age, sex, alcohol consumption status, status of ever smoking, level of education, physical exercise, prediabetes, family history of CVD, DBP, body mass index, serum concentrations of total cholesterol, high-density lipoproteins-C, glucose, uric acid and C-reactive protein, and eGFR, the cubic spline interpolation revealed that the relationship between different levels of baseline SBPs values within the normal range and the CVD risk showed a U-shaped curve, with the lowest point corresponding to a baseline SBP of $110 \mathrm{mmHg}$ (Fig. 1). However, statistically significant results were demonstrated only for SBP values $>125$ 
Table 1. Baseline characteristics of the study population.

\begin{tabular}{|c|c|c|c|c|c|}
\hline \multirow[b]{2}{*}{ Parameter } & \multicolumn{5}{|l|}{ SBP, $\mathbf{m m H g}$} \\
\hline & $\begin{array}{l}90-99 \\
(n=1548)\end{array}$ & $\begin{array}{l}100-109 \\
(n=4599)\end{array}$ & $\begin{array}{l}110-119 \\
(n=8136)\end{array}$ & $\begin{array}{l}120-129 \\
(n=11,246)\end{array}$ & $P$ for trend \\
\hline Age, years & $42.4 \pm 12.0$ & $44.4 \pm 12.2$ & $46.7 \pm 12.1$ & $49.5 \pm 12.1$ & $<0.001$ \\
\hline Men, $\mathrm{n}(\%)$ & $532(34.4)$ & $2118(46.1)$ & $4789(58.9)$ & $7858(69.9)$ & $<0.001$ \\
\hline $\mathrm{BMI}, \mathrm{kg} / \mathrm{m}^{2}$ & $22.7 \pm 3.16$ & $23.3 \pm 3.22$ & $24.0 \pm 3.25$ & $24.6 \pm 3.27$ & $<0.001$ \\
\hline $\mathrm{SBP}, \mathrm{mmHg}$ & $93.2 \pm 3.77$ & $103 \pm 3.72$ & $112 \pm 3.35$ & $122 \pm 2.83$ & $<0.001$ \\
\hline $\mathrm{LDL}, \mathrm{mg} / \mathrm{dL}$ & $80.0 \pm 28.2$ & $82.0 \pm 28.3$ & $85.1 \pm 29.8$ & $84.9 \pm 30.6$ & $<0.001$ \\
\hline $\mathrm{HDL}, \mathrm{mg} / \mathrm{dL}$ & $60.9 \pm 12.3$ & $60.7 \pm 12.7$ & $60.8 \pm 13.5$ & $61.4 \pm 14.0$ & 0.003 \\
\hline $\mathrm{Fbg}, \mathrm{mg} / \mathrm{dL}$ & $88.0 \pm 11.0$ & $88.5 \pm 11.1$ & $89.9 \pm 11.6$ & $91.1 \pm 11.9$ & $<0.001$ \\
\hline CRP, mg/L & $0.54(0.20,1.70)$ & $0.60(0.21,1.80)$ & $0.63(0.23 .1 .74)$ & $0.70(0.25,1.94)$ & 0.001 \\
\hline Exerciser, $n$ (\%) & $1398(93.1)$ & $4163(93.1)$ & 7377 (93.7) & $10145(94.4)$ & 0.013 \\
\hline Current drinker, $n(\%)$ & $326(21.5)$ & $997(22.1)$ & $1699(21.4)$ & $2119(19.6)$ & 0.001 \\
\hline Prediabetes, $n$ (\%) & $208(13.4)$ & $669(14.5)$ & $1524(18.7)$ & $2376(21.1)$ & $<0.001$ \\
\hline Family history of CVD, $n(\%)$ & $87(5.6)$ & $295(6.4)$ & $438(5.4)$ & $484(4.3)$ & $<0.001$ \\
\hline
\end{tabular}

SI conversion factors: To convert TC, HDL, and LDL to millimoles per liter, multiply by 0.0259 ; Fbg to millimoles per liter, multiply by 0.0555 ; SUA to micromoles per liter, divide by 0.0168 .

$B M I$ body mass index, SBP systolic blood pressure, $D B P$ diastolic blood pressure, $T G$ triglyceride, $T C$ total cholesterol, $L D L$ low-density lipoprotein, HDL highdensity lipoprotein, Fbg fasting blood glucose, CRP C-reactive protein, SUA serum uric acid, eGFR estimated glomerular filtration rate, CVD cardiovascular disease.

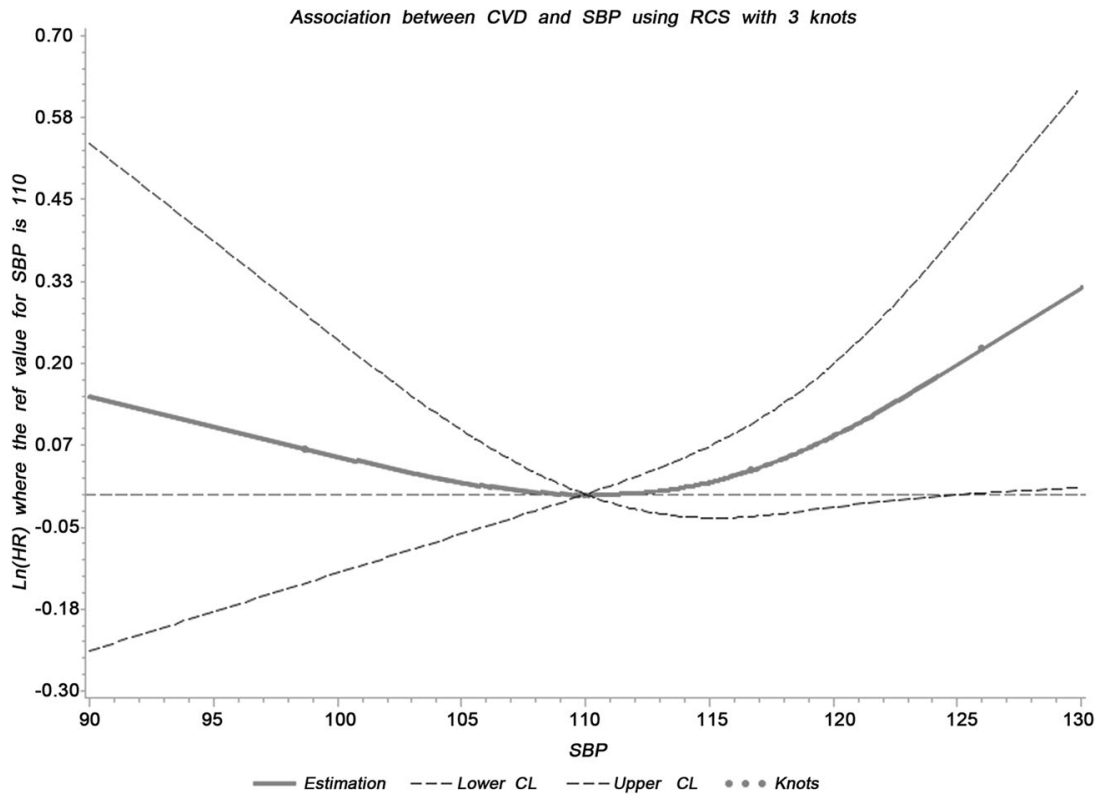

Fig. 1 Adjusted Cubic Spline for the Hazard of Incident Cardiovascular Disease (CVD) by Systolic Blood Pressure (SBP. Adjusted for age, sex (male/female), alcohol consumption status (never and past, current, $\geq 1$ time/day), ever smoking (yes/no), education level (elementary school, high school or above), exercise (none, occasionally or frequently, $\geq 1$ times/week), prediabetes (yes/no), family history of cardiovascular diseases (yes/no), diastolic blood pressure, body mass index, serum concentrations of total cholesterol, high-density lipoprotein cholesterol, glucose, uric acid and C-reactive protein, and estimated glomerular filtration rate.

$\mathrm{mmHg}$, while there was no statistical significance for SBP values $<125 \mathrm{mmHg}$ (Fig. 1). The categorical Cox regression further suggested that the CVD risk was not statistically significant for study participants with a baseline SBP level of $100-109 \mathrm{mmHg}$,
110-119 $\mathrm{mmHg}$, and $120-129 \mathrm{mmHg}$ compared with those with a baseline SBP level of $90-99 \mathrm{mmHg}$, after adjusting for the parameters listed above (Table 2; Supplementary Tables 1, 2). The Cox regression model also showed that there was no 
Table 2. Hazard ratios of cardiovascular events by systolic blood pressure categories within the normal range.

\begin{tabular}{|c|c|c|c|c|}
\hline \multirow[t]{2}{*}{ Parameter } & \multicolumn{4}{|c|}{ SBP, $\mathbf{m m H g}$} \\
\hline & 90-99 & 100-109 & 110-119 & $120-129$ \\
\hline Case/n & $24 / 1548$ & $106 / 4599$ & $265 / 8136$ & $452 / 11246$ \\
\hline Model 1 & Ref. & $1.48(0.95-2.30)$ & $2.10(1.38-3.18)^{\mathrm{a}}$ & $2.60(1.72-3.92)^{\mathrm{a}}$ \\
\hline Model 2 & Ref. & $1.20(0.77-1.88)$ & $1.43(0.94-2.18)$ & $1.45(0.96-2.19)$ \\
\hline Model 3 & Ref. & $1.12(0.69-1.80)$ & $1.21(0.75-1.93)$ & $1.09(0.67-1.77)$ \\
\hline \multicolumn{5}{|c|}{$\begin{array}{l}\text { Model 1: unadjusted. Model 2: adjusted for age and sex (male/female). Model 3: adjusted for age, sex (male/female), alcohol consumption status (never and } \\
\text { past, current, } \geq 1 \text { time/day), ever smoking (yes/no), education level (elementary school, high school or above), exercise (none, occasionally or frequently, } \geq 1 \\
\text { times/week), prediabetes (yes/no), family history of cardiovascular diseases (yes/no), diastolic blood pressure, body mass index, serum concentrations of total } \\
\text { cholesterol, high-density lipoprotein cholesterol, glucose, uric acid and C-reactive protein, and estimated glomerular filtration rate. Model } 4 \text { was a competing } \\
\text { risk model of death which adjusted for all the confounders in model 3. Compared with reference group. } \\
\text { a } P<0.01 \text {. }\end{array}$} \\
\hline
\end{tabular}

Table 3. Hazard ratios of cardiovascular events stratified by baseline systolic blood pressure categories in the normal range, after further excluding participants who had a DBP $\geq 80 \mathrm{mmHg}$.

\begin{tabular}{|c|c|c|c|c|}
\hline \multirow[t]{2}{*}{ Characteristic } & \multicolumn{4}{|c|}{ SBP, $\mathbf{m m H g}$} \\
\hline & 90-99 & $100-109$ & $110-119$ & $120-129$ \\
\hline Case/N & $24 / 1545$ & $96 / 4189$ & $161 / 5158$ & $108 / 2740$ \\
\hline Model 1 & Ref. & $1.47(0.94-2.30)$ & $2.01(1.31-3.09)^{\mathrm{a}}$ & $2.57(1.65-4.00)^{\mathrm{a}}$ \\
\hline Model 2 & Ref. & $1.23(0.78-1.92)$ & $1.35(0.88-2.07)$ & $1.19(0.76-1.87)$ \\
\hline Model 3 & Ref. & $1.22(0.75-1.99)$ & $1.28(0.78-2.11)$ & $1.06(0.62-1.79)$ \\
\hline \multicolumn{5}{|c|}{$\begin{array}{l}\text { Model 1: unadjusted. Model 2: adjusted for age and sex (male/female). Model 3: adjusted for age, sex (male/female), alcohol consumption status (never and } \\
\text { past, current, } \geq 1 \text { time/day), ever smoking (yes/no), education level (elementary school, high school or above), exercise (none, occasionally or frequently, } \geq 1 \\
\text { times/week), prediabetes (yes/no), family history of cardiovascular diseases (yes/no), diastolic blood pressure, body mass index, serum concentrations of total } \\
\text { cholesterol, high-density lipoprotein cholesterol, glucose, uric acid and C-reactive protein, and estimated glomerular filtration rate. Model } 4 \text { was a competing } \\
\text { risk model of death which adjusted for all the confounders in model } 3 \text {. Compared with reference group. } \\
\text { a } P<0.01 \text {. }\end{array}$} \\
\hline
\end{tabular}

significant interaction between age and SBP category and between sex and SBP category, so we did not perform further age or sex subgroup analysis.

The results remained overall unchanged if we additionally excluded participants with a DBP level $\geq 80 \mathrm{mmHg}$ or participants who had risk factor values outside the normal range (i.e., serum concentrations of low-density lipoproteins $-C \geq 130 \mathrm{mg} / \mathrm{dL}$, serum glucose concentration $\geq 100 \mathrm{mg} / \mathrm{dL}$, or women with a serum concentration of high-density lipoprotein-C level $<50 \mathrm{mg} / \mathrm{dL}$ ) (Tables 3, 4). If participants with a DBP level $\geq 80 \mathrm{mmHg}$ and participants with risk factor values outside the normal range were excluded, the associations between a baseline SBP of 100-109 $\mathrm{mmHg}, 110-119 \mathrm{mmHg}$, and $120-129 \mathrm{mmHg}$ and CVD incidence were still not significant (Table 5).

Finally, since participants with a normal SBP at baseline may develop hypertension during the follow-up, we entered the BP category as a time-dependent explanatory variable in a timedependent Cox regression analysis, in which at each point in time, the last available BP measurement was considered (Supplementary Tables 3, 4). In a similar manner, the parameter of intake of antihypertensive drugs and other covariates that changed over time were entered into the model as timedependent covariates. It revealed that the normal baseline SBP was still not associated with incident CVD, neither in the group of a baseline SBP of $120-129 \mathrm{mmHg}$ or in any other group. In addition, with a SBP level of $90-109 \mathrm{mmHg}$ as the reference, both the traditional and time-dependent Cox regression model suggested that the baseline SBP was not significantly associated with the incidence of CVDs.

\section{DISCUSSION}

The current study found that the CVD incidence increased with higher baseline SBP level in the normotensive population without hypertension and without other cardiovascular risk factors at baseline. After adjusting for risk factors, the relationship between higher baseline SBP (within the normal range) and higher CVD risk showed a U-shaped curve. However, statistically significant results were demonstrated only for SBP values $>125 \mathrm{mmHg}$, while there was no statistical significance for SBP values $<125 \mathrm{mmHg}$. The categorical Cox regression further suggested that the CVD risk was not statistically significant for study participants with a baseline SBP level of $100-109 \mathrm{mmHg}, 110-119 \mathrm{mmHg}$, and $120-129 \mathrm{mmHg}$ compared with those with a baseline SBP level of $90-99 \mathrm{mmHg}$, after adjusting for the parameters listed above.

In contrast to our study, the MESA reported on a dose-effect relationship between baseline SBP level and CVD risk in a population without hypertension and without other cardiovascular risk factors [8]. The reasons for the discrepancy between both studies may be differences in the study population, sample size ( $n$ $=1457$ versus $n=25,529$ ), age (mean age 58 years versus 47 years), and rate of CVD events (6.5\% (MESA) versus $2.7 \%$ in our study) and the inclusion of BP measurements and other CVD risk factors, examined during the follow-up period, into the statistical 
Table 4. Hazard ratios of cardiovascular events stratified by baseline normal systolic blood pressure categories in the normal range, after further excluding participants who had risk factor values above normal (i.e., serum concentrations of low-density lipoproteins-C $\geq 130 \mathrm{mg} / \mathrm{dL}$, serum glucose concentration $\geq 100 \mathrm{mg} / \mathrm{dL}$, or women with a serum concentration of high-density lipoprotein-C level $<50 \mathrm{mg} / \mathrm{DI}$ ).

\begin{tabular}{|c|c|c|c|c|}
\hline \multirow[t]{2}{*}{ Characteristic } & \multicolumn{4}{|c|}{$\mathrm{SBP}, \mathrm{mmHg}$} \\
\hline & 90-99 & 100-109 & $110-119$ & $120-129$ \\
\hline Case/N & $21 / 1158$ & $76 / 3407$ & $195 / 5790$ & $305 / 7888$ \\
\hline Model 1 & Ref & $1.22(0.75-1.98)$ & $1.86(1.18-2.91)^{\mathrm{a}}$ & $2.14(1.37-3.32)^{\mathrm{a}}$ \\
\hline Model 2 & Ref & $1.00(0.62-1.62)$ & $1.29(0.82-2.03)$ & $1.20(0.77-1.88)$ \\
\hline Model 3 & Ref & $0.90(0.53-1.52)$ & $1.10(0.65-1.85)$ & $0.89(0.52-1.53)$ \\
\hline \multicolumn{5}{|c|}{$\begin{array}{l}\text { Model 1: unadjusted. Model 2: adjusted for age and sex (male/female). Model } 3 \text { : adjusted for age, sex (male/female), alcohol consumption status (never and } \\
\text { past, current, } \geq 1 \text { time/day), ever smoking (yes/no), education level (elementary school, high school or above), exercise (none, occasionally or frequently, } \geq 1 \\
\text { times/week), family history of cardiovascular diseases (yes/no), diastolic blood pressure, body mass index, serum concentrations of total cholesterol, high- } \\
\text { density lipoprotein cholesterol, glucose, uric acid and C-reactive protein, and estimated glomerular filtration rate. Model } 4 \text { was a competing risk model of } \\
\text { death which adjusted for all the confounders in model } 3 \text {. Compared with reference group. } \\
{ }^{a} P<0.01 \text {. }\end{array}$} \\
\hline
\end{tabular}

analysis in our study. In other study populations, a baseline SBP between 120 and $129 \mathrm{mmHg}$ as compared with normal SBP (SBP $<120 \mathrm{mmHg}$ ) increased the CVD risk, however, traditional cardiovascular risk factors such as diabetes and dyslipidemia were not fully excluded [21-24].

Since an association between isolated diastolic hypertension and incident CVD has not unequivocally been confirmed, participants with a DBP $\geq 80 \mathrm{mmHg}$ were not excluded in the initial analysis in our study $[25,26]$. Interestingly, the results of our analysis did not markedly change after excluding study participants with a DBP $\geq 80 \mathrm{mmHg}$ in a sensitivity analysis. In a similar manner, when we excluded participants with diabetes or dyslipidemia, the results of the analysis did not change. When we excluded both, individuals with isolated diastolic hypertension and participants with other cardiovascular risk factors, the adjusted HR for CVD was neither statistically significant (Table 3). One of the reasons for that finding may have been the reduction in the number of CVD events limiting the statistical power of the analysis.

We also observed that the baseline SBP was not correlated with the CVD incidence when the BP category as a time-dependent explanatory variable was included in a time-dependent Cox regression analysis. However, the proportion of participants who eventually progressed to hypertension gradually increased with the baseline SBP categories. Correspondingly, the levels of traditional cardiovascular risk factors such as body mass index, serum concentrations of total cholesterol, low-density lipoproteinC, glucose, C-reactive protein and uric acid, increased with higher baseline SBP within the normal range. It suggests that individuals with elevated SBP should be monitored closely for the eventual development of CVD risk factors which may then cause an increased incidence of CVD. It holds true in particular since studies have shown that the cumulative effects of these CVD risk factors increase the CVD risk [27-29].

The clinical implications of results of our study are first, that a SBP between 90 and $129 \mathrm{mmHg}$ is not associated with an increased CVD risk in a healthy population. Second, even in the healthy population, some traditional cardiovascular risk factors still presented a trend of aggregation with the increase of baseline SBP level. Therefore, we advise prevention and control of cardiovascular risk factors through dietary and lifestyle improvements to reduce the cardiovascular risk in the healthy population.

When the results of our study are discussed, its limitations should be taken into account. First, our study was not an intervention trial and has just shown associations. Second, although the participants with a SBP of $120-129 \mathrm{mmHg}$ did not have any medical problems, they showed a trend toward CVD risk factors, what might have influenced the development of CVDs (Table 1). Third, we did not include all cardiovascular risk factors, such as high-salt diet and air pollution, into our analysis. Fourth, the CVD rate in our study population was lower than in the MESA study population, perhaps due to that our study participants were mainly employed, had a younger age and that the follow-up was shorter. The CVD rate might therefore have been underestimated in our study. Fifth, in agreement with the MESA study, the proportion of women decreased and the mean age increased with higher SBP category. Since the CVD incidence depends on age and sex, the uneven distribution of both parameters might have led to a bias. Further statistical analysis however did not show a significant interaction between age or sex and the SBP categories. Sixth, the individuals with a baseline SBP of $120-129 \mathrm{mmHg}$ were older than those with a baseline SBP of $90-110 \mathrm{mmHg}(49.5 \pm 12.1$ years versus $42.4 \pm 12.0$ years; $P<0.001$ ), so that one may argue that this age difference at the baseline examination and not the $\mathrm{BP}$ might have been responsible for the noted outcomes. In the multivariable analysis, however, age was included into the list of independent variables, and the results remained to be statistically significant. Strengths of our study include the study sample size, the relatively high number of parameters and risk factors assessed, the repeated measures of the CVD risk factors, the standardized data collection protocols, and a mostly complete follow-up for the detection of CVD events, since the Municipal Social Insurance collected all medical records of the entire population of the Kailuan community.

In conclusion, in this population without hypertension and without other traditional cardiovascular risk factors at baseline, although SBP levels were not found to be associated with CVD risk, individuals with an elevated baseline SBP level of 120-129 $\mathrm{mmHg}$ had indirectly a higher CVD risk through a higher chance of developing cardiovascular risk factors such as hypertension. Individual with a high-normal SBP of $120-129 \mathrm{mmHg}$ may be closely followed for an early detection of a BP rise as main risk factor for incident CVDs.

\section{Summary}

What is known about the topic

- High BP as an important risk factor for CVDs is a leading cause of disability and death. It has remained unclear whether the association between the SBP level and the CVD risk is valid also among healthy normotensive adults without traditional cardiovascular risk factors. 
Table 5. Hazard ratios of cardiovascular events stratified by baseline systolic blood pressure categories in the normal range, after excluding participants who had a diastolic blood pressure $\geq 80 \mathrm{mmHg}$ and had risk factor values above normal (i.e., serum concentrations of low-density lipoproteins- $\mathrm{C} \geq 130 \mathrm{mg} / \mathrm{dL}$, serum glucose concentration $\geq 100 \mathrm{mg} / \mathrm{dL}$, or women with a serum concentration of high-density lipoprotein-C level < $50 \mathrm{mg} / \mathrm{Dl})$.

\section{Characteristic}

Case/N

Incidence/1000 person-years

Model 1

Model 2

Model 3

Model 4

\begin{tabular}{ll}
$\mathbf{S B P}, \mathbf{m m H g}$ & \\
\hline $\mathbf{9 0 - 9 9}$ & $\mathbf{1 0 0 - 1 0 9}$ \\
\hline $21 / 1156$ & $68 / 3121$ \\
\hline 1.70 & 2.03 \\
\hline Ref. & $1.19(0.73-1.95)$ \\
\hline Ref. & $1.00(0.61-1.63)$ \\
\hline Ref. & $0.98(0.57-1.70)$ \\
\hline Ref. & $1.02(0.58-1.78)$
\end{tabular}

$110-119$
$116 / 3667$
2.98
$1.74(1.10-2.77)^{b}$
$1.19(0.74-1.89)$
$1.19(0.68-2.07)$
$1.22(0.69-2.16)$

120-129

$73 / 1941$

3.57

$2.09(1.29-3.39)^{\mathrm{a}}$

$0.95(0.58-1.55)$

$0.88(0.48-1.59)$

$0.91(0.50-1.68)$

Model 1: unadjusted. Model 2: adjusted for age and sex (male/female). Model 3: adjusted for age, sex (male/female), alcohol consumption status (never and past, current, $\geq 1$ time/day), ever smoking (yes/no), education level (elementary school, high school or above), exercise (none, occasionally or frequently, $\geq 1$ times/week), family history of cardiovascular diseases (yes/no), diastolic blood pressure, body mass index, serum concentrations of total cholesterol, highdensity lipoprotein cholesterol, glucose, uric acid and C-reactive protein, and estimated glomerular filtration rate. Model 4 was a competing risk model of death which adjusted for all the confounders in model 3 . Compared with reference group.

${ }^{\mathrm{a}} P<0.01$.

${ }^{\mathrm{b}} P<0.05$.

- The MESA reported that normotensive participants without conventional cardiovascular risk factors showed during an average follow-up of 14.5 years an increase in the CVD risk by $53 \%$ for every $10 \mathrm{mmHg}$ increase in the baseline SBP. It suggested a dose-effect rather than a J-shaped or U-shaped relationship between the SBP level and CVD risk in a healthy population.

- Since risk factors differ between regions and societies, we performed this study to investigate the association between the baseline SBP level within the normal range and the CVD risk in a normotensive population without conventional cardiovascular risk factors in China.

\section{What this study adds}

- In this cohort study including 25,529 participants without CVD at baseline (follow-up: $10.6 \pm 1.5$ years), the CVD incidence per 1000 person-years increased in univariate analysis with higher baseline SBP levels within the normotensive range.

- After adjusting for CVD risk factors, the CVD risk was not statistically significant for normotensive study participants with a baseline SBP level of $100 \mathrm{mmHg}$ or higher as compared with those with a baseline SBP level of $90-99 \mathrm{mmHg}$.

- As a corollary, if CVD risk factors including BP categories which developed during follow-up were included in a timedependent Cox regression analysis, the normal baseline SBP was neither associated with incident CVDs.

\section{REFERENCES}

1. GBD 2019 Risk Factors Collaborators. Global burden of 87 risk factors in 204 countries and territories, 1990-2019: a systematic analysis for the Global Burden of Disease Study 2019. Lancet. 2020;396:1223-49.

2. Roth GA, Mensah GA, Johnson CO, Addolorato G, Ammirati E, Baddour LM, et al. Global burden of cardiovascular diseases and risk factors, 1990-2019: update from the Global Burden of Disease 2019 Study. J Am Coll Cardiol. 2020;76:2982-3021.

3. Forouzanfar MH, Liu P, Roth GA, Ng M, Biryukov S, Marczak L, et al. Global burden of hypertension and systolic blood pressure of at least 110 to $115 \mathrm{~mm} \mathrm{Hg}, 1990-$ 2015. JAMA. 2017;317:165-82.
4. Tajeu GS, Booth JN, Colantonio LD, Gottesman RF, Howard G, Lackland DT, et al. Incident cardiovascular disease among adults with blood pressure $<140 / 90 \mathrm{~mm}$ Hg. Circulation. 2017;136:798-812.

5. Lewington S, Clarke R, Qizilbash N, Peto R, Collins R, Prospective Studies Collaboration. Age specific relevance of usual blood pressure to vascular mortality: a meta-analysis of individual data for one million adults in 61 prospective studies. Lancet. 2002;360:1903-13.

6. SPRINT Research Group, Wright JT Jr, Williamson JD, Whelton PK, Snyder JK, Sink $\mathrm{KM}$, et al. A Randomized trial of intensive versus standard blood-pressure control. N Engl J Med. 2015;373:2103-16.

7. Whelton PK, Carey RM, Aronow WS, Casey DE Jr, Collins KJ, Dennison Himmelfarb C, et al. 2017 ACC/AHA/AAPA/ABC/ACPM/AGS/ APhA/ASH/ASPC/NMA/PCNA Guideline for the prevention, detection, evaluation, and management of high blood pressure in adults: a report of the American College of Cardiology/American Heart Association task force on clinical practice guidelines. Hypertension. 2018;71:e13-e115.

8. Whelton SP, McEvoy JW, Shaw L, Psaty BM, Lima JAC, Budoff M, et al. Association of normal systolic blood pressure level with cardiovascular disease in the absence of risk factors. JAMA Cardiol. 2020;5:1011-8.

9. Wang C, Yuan Y, Zheng M, Pan A, Wang M, Zhao M, et al. Association of age of onset of hypertension with cardiovascular diseases and mortality. J Am Coll Cardiol. 2020;75:2921-30.

10. Wu S, An S, Li W, Lichtenstein AH, Gao J, Kris-Etherton PM, et al. Association of trajectory of cardiovascular health score and incident cardiovascular disease. JAMA Netw Open. 2019;2:e194758.

11. Wu S, Song Y, Chen S, Zheng M, Ma Y, Cui L, et al. Blood pressure classification of 2017 associated with cardiovascular disease and mortality in young Chinese adults. Hypertension. 2020;76:251-8.

12. Levey AS, Stevens LA, Schmid CH, Zhang YL, Castro AF 3rd, Feldman $\mathrm{HI}$, et al. A new equation to estimate glomerular filtration rate. Ann Intern Med. 2009;150:604-12.

13. Grundy SM, Stone NJ, Bailey AL, Beam C, Birtcher KK, Blumenthal RS, et al. AHA/ ACC/AACVPR/AAPA/ABC/ACPM/ ADA/ AGS/APhA/ASPC/NLA/PCNA Guideline on the management of blood cholesterol: executive summary-a report of the American College of Cardiology/American Heart Association task force on clinical practice guidelines. J AmColl Cardiol. 2019;73:3168-209.

14. Arnett DK, Blumenthal RS, Albert MA, Buroker AB, Goldberger ZD, Hahn EJ, et al. 2019 ACC/AHA Guideline on the primary prevention of cardiovascular disease: a report of the American College of Cardiology/American Heart Association task force on clinical practice guidelines. Circulation. 2019;140:e596-e646.

15. Fernández-Friera L, Fuster V, López-Melgar B, Oliva B, García-Ruiz JM, Mendiguren $\mathrm{JH}$, et al. Normal LDL-cholesterol levels are associated with subclinical atherosclerosis in the absence of risk factors. J AmColl Cardiol. 2017;70:2979-91.

16. Jin C, Chen S, Vaidya A, Wu Y, Wu Z, Hu FB, et al. Longitudinal change in fasting blood glucose and myocardial infarction risk in a population without diabetes. Diabetes Care. 2017;40:1565-72. 
17. Tunstall-Pedoe H, Kuulasmaa K, Amouyel P, Arveiler D, Rajakangas AM, Pajak A. Myocardial infarction and coronary deaths in the World Health Organization MONICA Project: registration procedures, event rates, and case-fatality rates in 38 populations from 21 countries in four continents. Circulation. 1994;90:583-612.

18. Heart Failure Group of Chinese Society of Cardiology of Chinese Medical Association; Chinese Heart Failure Association of Chinese Medical Doctor Association; Editorial Board of Chinese Journal of Cardiology. Chinese guidelines for the diagnosis and treatment of heart failure 2018. Zhonghua Xin Xue Guan Bing Za Zhi. 2018;46:760-89.

19. Stroke-1989: recommendations on stroke prevention, diagnosis, and therapyreport of the. WHO Task Force on Stroke and other Cerebrovascular Disorders. Stroke. 1989;20:1407-31.

20. Harrell FE. Regression modeling strategies: with applications to linear models, logistic regression, and survival analysis. Heidelberg:Springer; 2001.

21. Son JS, Choi S, Kim K, Kim SM, Choi D, Lee G, et al. Association of blood pressure classification in Korean young adults according to the 2017 American College of Cardiology/American Heart Association guidelines with subsequent cardiovascular disease events. JAMA. 2018;320:1783-92.

22. Yano Y, Reis JP, Colangelo LA, Shimbo D, Viera AJ, Allen NB, et al. Association of blood pressure classification in young adults using the 2017 American College of Cardiology/American Heart Association blood pressure guideline with cardiovascular events later in life. JAMA. 2018;320:1774-82.

23. Jung MH, Yi SW, An SJ, Yi JJ. Age-specific associations between systolic blood pressure and cardiovascular mortality. Heart. 2019;105:1070-7.

24. Kim S, Chang Y, Kang J, Cho A, Cho J, Hong YS, et al. Relationship of the blood pressure categories, as defined by the ACC/AHA 2017 blood pressure guidelines, and the risk of development of cardiovascular disease in low-risk young adults: insights from a retrospective cohort of young adults. J Am Heart Assoc. 2019;8: e011946.

25. McEvoy JW, Daya N, Rahman F, Hoogeveen RC, Blumenthal RS, Shah AM, et al Association of Isolated Diastolic Hypertension as Defined by the 2017 ACC/AHA Blood Pressure Guideline With Incident Cardiovascular Outcomes. JAMA. 2020;323:329-38.

26. Wu S, Ji C, Shi J, Chen S, Huang Z, Jonas JB. Isolated diastolic hypertension as defined by the 2017 American College of Cardiology/American Heart Association blood pressure guideline and incident cardiovascular events in Chinese. J Hypertens. 2021;39:519-25.

27. Wang A, Liu J, Li C, Gao J, Li X, Chen S, et al. Cumulative exposure to highsensitivity C-reactive protein predicts the risk of cardiovascular disease. J Am Heart Assoc. 2017;6:e005610.

28. Domanski MJ, Tian X, Wu CO, Reis JP, Dey AK, Gu Y, et al. Time course of LDL cholesterol exposure and cardiovascular disease event risk. J Am Coll Cardiol. 2020;76:1507-16.

29. Zhang $Y$, Vittinghoff $E$, Pletcher MJ, Allen NB, Al Hazzouri AZ, Yaffe $K$, et al. Associations of blood pressure and cholesterol levels during young adulthood with later cardiovascular events. J Am Coll Cardiol. 2019;74:330-41.

\section{AUTHOR CONTRIBUTIONS}

Design of the study: CJ, NW, JS, ZH, SC, GW, SW; Measurement and collection of the data: CJ, NW, JS, ZH, SC, GW, SW; Statistical analysis: CJ, NW, JS, ZH, SC, GW, SW, JBJ; Writing the first draft of the paper: $\mathrm{CJ}, \mathrm{NW}, \mathrm{JS}, \mathrm{ZH}, \mathrm{SC}, \mathrm{GW}, \mathrm{SW}, \mathrm{JBJ}$; Amendment of the paper and final approval: CJ, NW, JS, ZH, SC, GW, SW, JBJ.

\section{FUNDING}

Open Access funding enabled and organized by Projekt DEAL.

\section{COMPETING INTERESTS}

The authors declare no competing interests.

\section{ADDITIONAL INFORMATION}

Supplementary information The online version contains supplementary material available at https://doi.org/10.1038/s41371-021-00598-1.

Correspondence and requests for materials should be addressed to Shouling $\mathrm{Wu}$ or Jost B. Jonas.

Reprints and permission information is available at http://www.nature.com/ reprints

Publisher's note Springer Nature remains neutral with regard to jurisdictional claims in published maps and institutional affiliations.

(i) Open Access This article is licensed under a Creative Commons cc) Attribution 4.0 International License, which permits use, sharing, adaptation, distribution and reproduction in any medium or format, as long as you give appropriate credit to the original author(s) and the source, provide a link to the Creative Commons license, and indicate if changes were made. The images or other third party material in this article are included in the article's Creative Commons license, unless indicated otherwise in a credit line to the material. If material is not included in the article's Creative Commons license and your intended use is not permitted by statutory regulation or exceeds the permitted use, you will need to obtain permission directly from the copyright holder. To view a copy of this license, visit http://creativecommons. org/licenses/by/4.0/.

(c) The Author(s) 2021 